\title{
Fractal morphological analysis of Bacteriorhodopsin (bR) layers deposited onto Indium Tin Oxide (ITO) electrodes
}

\author{
P. Vengadesh *, S.V. Muniandy, W.H. Abd. Majid \\ Department of Physics, Faculty of Science, University of Malaya, Pantai Valley, 50603 Kuala Lumpur, Malaysia
}

\section{A R T I C L E I N F O}

\section{Article history:}

Received 29 July 2008

Received in revised form 14 October 2008

Accepted 23 December 2008

Available online 6 January 2009

\section{Keywords:}

Bacteriorhodopsin thin films

Organic optoelectronic material

Coffee-ring effect

Fractal analysis

\begin{abstract}
A B S T R A C T
Uniform Bacteriorhodopsin layers for the purpose of fabricating Bacteriorhodopsin-based biosensors were prepared by allowing drying of the layers under a constant electric field. To properly observe and understand the "electric field effect" on the protein Bacteriorhodopsin, the electric and non-electric field influenced Bacteriorhodopsin layers prepared using a manual syringe-deposition method applied onto Indium Tin Oxide electrodes were structurally investigated using Scanning Electron Microscopy and Atomic Force Microscopy. The results yield obvious morphological differences between the electric and non-electric field assisted Bacteriorhodopsin layers and brings to attention the occurrence of the so-called "coffee-ring" effect in the latter case. We applied stochastic fractal method based on the generalized Cauchy process to describe the morphological features surrounding the void. Fractal dimension is used to characterize the local regularity of the Bacteriorhodopsin clusters and the correlation exponent is used to describe the long-range correlation between the clusters. It is found that the Bacteriorhodopsin protein tends to exhibit with strong spatial correlation in the presence of external electric field compared to in absence of the electric field. Long-range correlation in the morphological feature may be associated to the enhancement of aggregation process of Bacteriorhodopsin protein in the presence of electric field, thereby inhibiting the formation of the so-called "coffee-ring" effect. As such, the observations discussed in this work suggest some amount of control of surface uniformity when forming layers.
\end{abstract}

(c) 2009 Elsevier B.V. All rights reserved.

\section{Introduction}

The biomaterial Bacteriorhodopsin (bR) is a photosensitive protein studied extensively since its photo-induced proton-pumping properties in nature was discovered almost four decades ago by Oesterhelt [1]. It is synthesized by an extremely halophilic bacterium found thriving in salt marshes as an alternate way to produce Adenosine TriPhosphate (ATP) from Adenosine Dwi-Phosphate (ADP) during anaerobic respiration. Under conditions of extremely low $\mathrm{O}_{2}$ concentrations, the bR is produced in patches [2] known as the Purple Membrane (PM) by the bacterium to harness the sunlight in a photosynthesis process known as the photocycle process. Upon light absorption, the protein undergoes various complex structural and conformational changes that involve the transportation of protons or charge movements from the intracellular to the extracellular regions of the PM [3]. The unidirectional charge movement in turn generates a light dependent photocurrent that earns bR the importance of being a suitable photosensitive bio-molecular material in various bio-devices fabricated by many groups worldwide [4-7] and as shown in our earlier work on bR-Carboxymethylcellulose (CMC) photosensors [8].

\footnotetext{
* Corresponding author. Tel.: +60 379674038; fax: +60 379674146

E-mail address: vengadeshp@hotmail.com (P. Vengadesh).
}

The concentrated research into preparing bR thin films to be incorporated into the construction of various bR-based sensors basically involves the Langmuir-Blodgett (LB) method of film

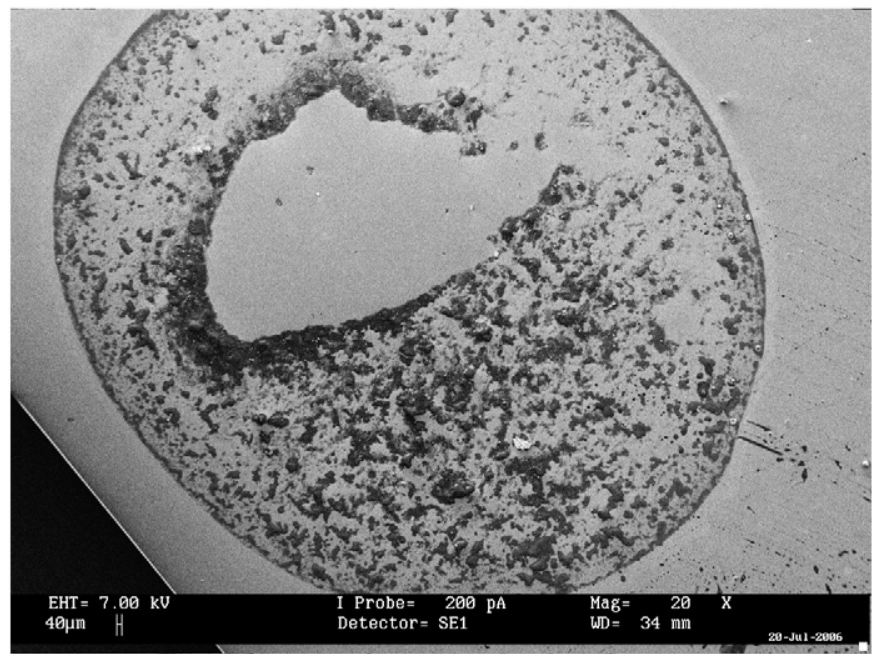

Fig. 1. 15 $\times$ magnifications of non-electric field assisted bR film showing a void center. 
preparation $[4,9,10]$. While not downplaying the advantages of using the LB method of preparing mono and multilayer bR films, an easier and less tedious approach was applied in our earlier work on fabricating the bR-CMC photosensor. The so-called "coffee-ring" effect was also observed in our bR films formed using the manual syringedeposition technique [8] as with many other air-dried organic material films. The resulting effect characterized by concentration of the majority of the material residue along the perimeter of the ring indicates fundamental reactions since the traditional mechanism of solute transport such as the Marangoni flow [11,12], Rayleigh-Bernard processes or the wetting phenomenon cannot explain the existence of the ring. The fundamental process that governs the "coffee-ring" effect was later attributed to a previously unknown form of capillary flow. According to Deegan et al. $[13,14]$, the pinned contact line of the

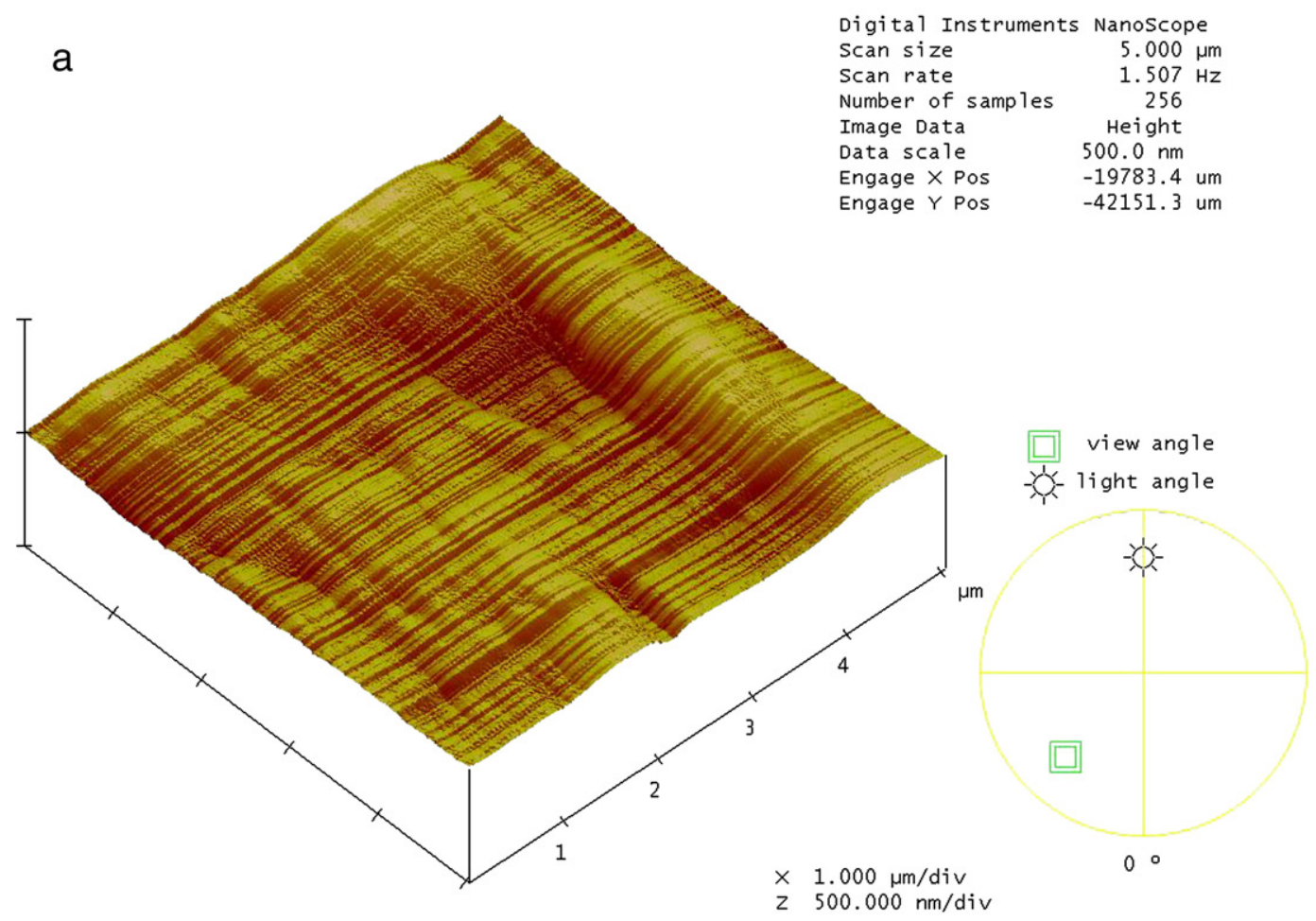

3 dabr2

b

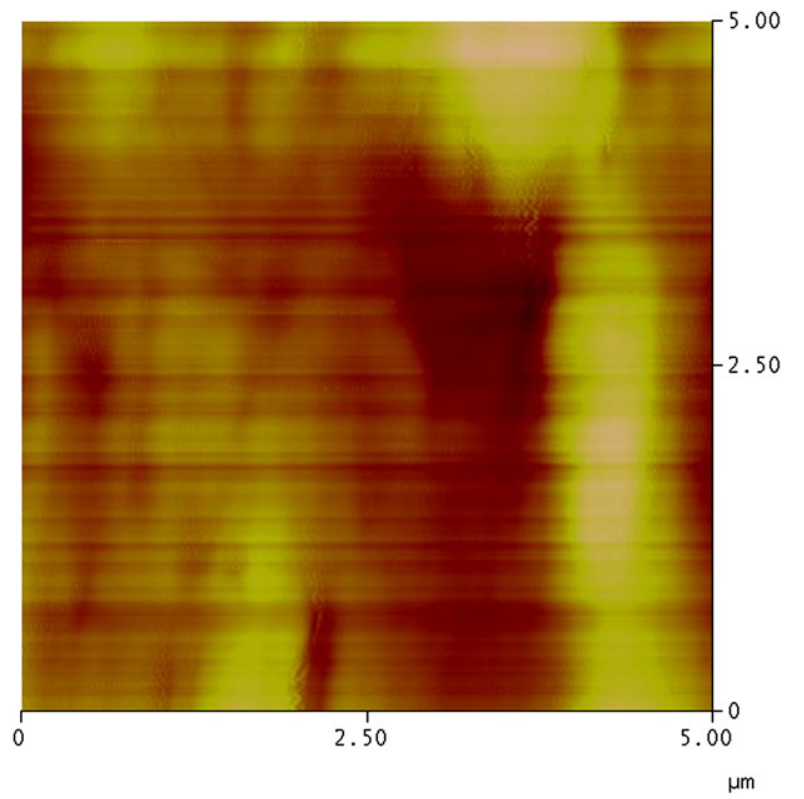

Digital Instruments Nanoscope $\quad 5.000 \mu \mathrm{m}$ $1.507 \mathrm{~Hz}$ Number of samples 256 age Data Engage $X$ Pos $\quad-19783.4$ um 42151.3 um 
drying drop allows evaporation of liquid from the edge of the ring while being replenished by liquid from the interior. As such, the resulting capillary flow as the droplet dries through the edge of the ring forces the material existing in the centre in an outward flow leading to the rim of the ring. While this phenomenon seems favorable in many applications, this mechanism does not allow uniform formations of most organic films for the study of organic thin films under normal room conditions since the centre of the film always remain as a void. Thus, a practical method of inhibiting or reversing the "coffee-ring" effect using a constant electric field was applied in our work. As will be shown, bR thin films dried under the influence of a constant electric field resulted in a visible uniform surface layer and clearly did not yield any coffee-ring stains.

In our present work, we investigated the resulting morphological surface changes between electric and non-electric field induced bR layers formed on the Indium Tin Oxide (ITO) conductive slides. Surface morphology observations carried-out through the utilization of the Scanning Electron Microscope (SEM) and Atomic Force Microscope (AFM) images enabled two and three-dimensional visualization of the films' surface respectively. The images gathered clearly show the ability of the electric field to reverse the "coffee-ring" effect and therefore indicates some amount of qualitative control of surface uniformity in bR layers. Although SEM images complemented by AFM imaging of the bR membranes and films have been carried-out elsewhere $[15,16]$, the occurrence of the "coffee-ring" effect has not be sufficiently studied in bR layers. In these work, we applied a stochastic fractal model, namely the generalized Cauchy process [17] to characterize the spatial distribution of bR proteins sampled in the region around the coffee ring. Among the most commonly used random surface models are the two-dimensional Brownian motion and the fractional Brownian motion (FBM) [18]. The main advantage of using generalized Cauchy process (GCP) compared to FBM is that the former allows simultaneous but independent description of the fractal as well as the long-range correlation behaviors. Time domain GCP has been recently used for modeling relaxation phenomena by Lim and $\mathrm{Li}$ [19] and its applications for material surfaces modeling is given in the work by Tscheschel et al. [20]. A rather special GCP type process with fixed fractal characteristic was studied by Maske et al. [21] with application to percolation clusters. In this paper, we showed that the two parameters GCP is useful for morphological modeling of bR protein cluster distributions.

\section{Experiment}

\subsection{Material preparation}

PM (molecular weight without retinal of $26784 \mathrm{Da}$ ) of variant type V1 containing purple-coloured lyophilized powder of bR molecules isolated from Halobacterium salinarium were obtained from MIB, (Munich Innovative Biomaterials), Germany. Product information stated absorption maximum of bR in deionized water after light adaptation at $566 \mathrm{~nm}$ while absorbance ratio (referring to the ratio of maximum optical densities, $\mathrm{ODs}$ at $280 \mathrm{~nm}$ and $570 \mathrm{~nm}$ ), $\mathrm{OD}_{280} / \mathrm{OD}_{570}$ at 2,3. A bR concentration of $0.2 \mathrm{mg} \mathrm{ml}^{-1}$ were achieved by suspending bR crystals in deionized water and the resulting suspension agitated with an agitator (Model 34524, supplied by Snijders Scientific, Holland). Due to bR's low solubility in water, the bR suspension was agitated vigorously for short periods of time for three days. Storage, in between the days of preparation was at normal freezing conditions in a dark environment. The bR suspension should only be agitated for short periods each day since prolonged agitation or sonication might destroy the fragile molecular structure of bR. As such, only about 20 minutes of agitation consisting of 5 minutes of low speed followed by 10 minutes of high speed and another 5 minutes of low speed agitation was allowed on day one and day two. After two days of agitation, countless fine particles were still observed against a bright background suggesting incomplete solubility of the bR particles in the deionized water. Uniform light purple colouration seen right after agitation due to uniform distribution of the purple-coloured bR molecules or PM separates into two parts when the darker-coloured bR molecules settles when left undisturbed. Finally on day three, the suspension was again agitated for 20 minutes but at 10 minutes of low and high speeds each resulting in an almost particles-free bR solution. The resulting solution exhibited an almost uniform dark-purplish solution as a result of the high bR solubility achieved through the process of agitation. The semi-transparent ITO slide with dimensions of $(2.5 \mathrm{~cm} \times 7.7 \mathrm{~cm})$ meanwhile had a surface resistivity of about $(75.0 \pm 0.1) \Omega \mathrm{cm}^{-1}$.

\subsection{Experimental setup}

A drop of bR solution, about $0.1 \mathrm{ml}$ applied from a $1.0 \mathrm{ml}$ syringe was deposited directly onto the ITO glass slides. For the electric assisted thin film formation, the working electrode bearing the bR solution was placed on an Aluminium base (positively biased with a direct current source) and kept under a high electric field of $50.0 \mathrm{~V} \mathrm{~cm}^{-1}$ to induce high molecular orientation of the bR molecules. Earlier published work by the author [8] in the same laboratory has clearly shown the non-depreciation of photoresponse when using bR-sensors with bR films fabricated at the same electric field intensity as applied to the present work. As such, it could be concluded that the bR protein does not undergo the denaturization process when subjected to the electric field of about $50 \mathrm{~V} \mathrm{~cm}^{-1}$. Other researchers, such as Kononenko et al., 1987 [22], have also carried out investigations into field-related effects on the protein. It has been shown in the latter work that higher electric fields do have a degrading effect on the functionality of the protein. However, the degrading effect is negligible at the present value of electric field utilized in this study. This was achieved by utilising the proteins' natural net negative charge and electric dipole moment to produce high orientation of the bR molecules even after drying [23]. Silver paint with a resistivity of about $(1.2 \pm 0.1) \Omega \mathrm{cm}^{-1}$, connecting the ITO electrode to the underside of the glass slide bearing the bR solution enables electrical connection to the aluminium base. Both the electric and non-electric assisted films were kept in dark for about 48 hours to allow drying at normal room temperature. Large view Scanning Electron Microscope investigations carried-out were conducted using a LEICA Stereoscan S440 Digital Scanning Electron Microscope.

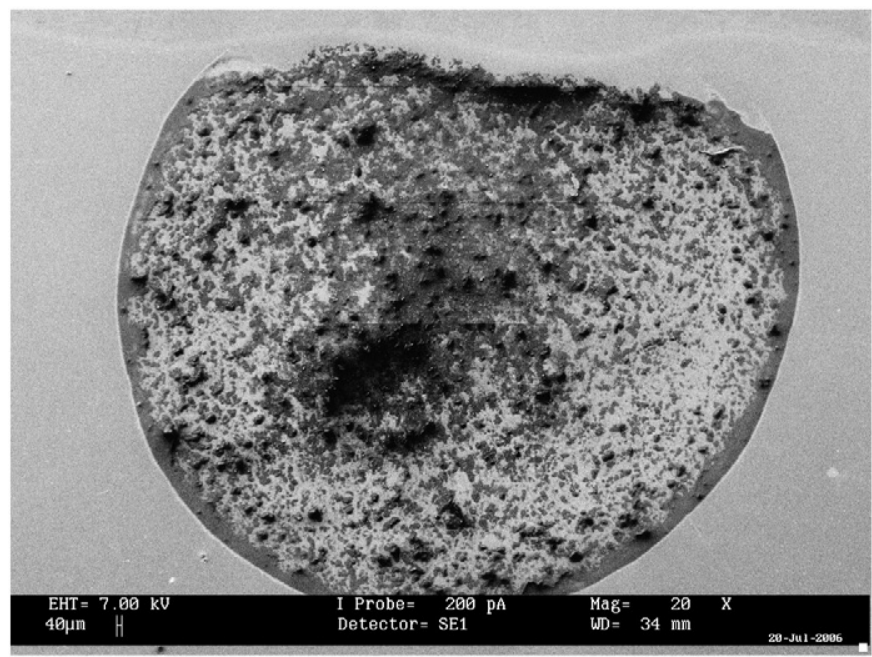

Fig. 3. 15 $\times$ magnifications of the electric field assisted bR film showing the centralized and compressed region. 


\section{Results and discussions}

\subsection{Morphological comparison}

Observations on the bR layers (15X magnification) showed some distinct and interesting features. The non-electric field assisted bR layer always had a centre void of any material as shown in Photomicrograph 1. The same profile was observed in other non- electric field assisted bR samples prepared in the same manner. As can be easily seen from the SEM, the bR concentrated ring edge clearly exhibits the "coffee-ring" effect. Meanwhile, the AFM pictures shown in Picture 1 further illustrate the central void effect in terms of surface topology. The depth profile of the pictures clearly indicates minimal or almost zero deposition in the centre allowing a certain "outwarddirected" force that expels materials towards the edge of the ring (Figs. 1 and 2).

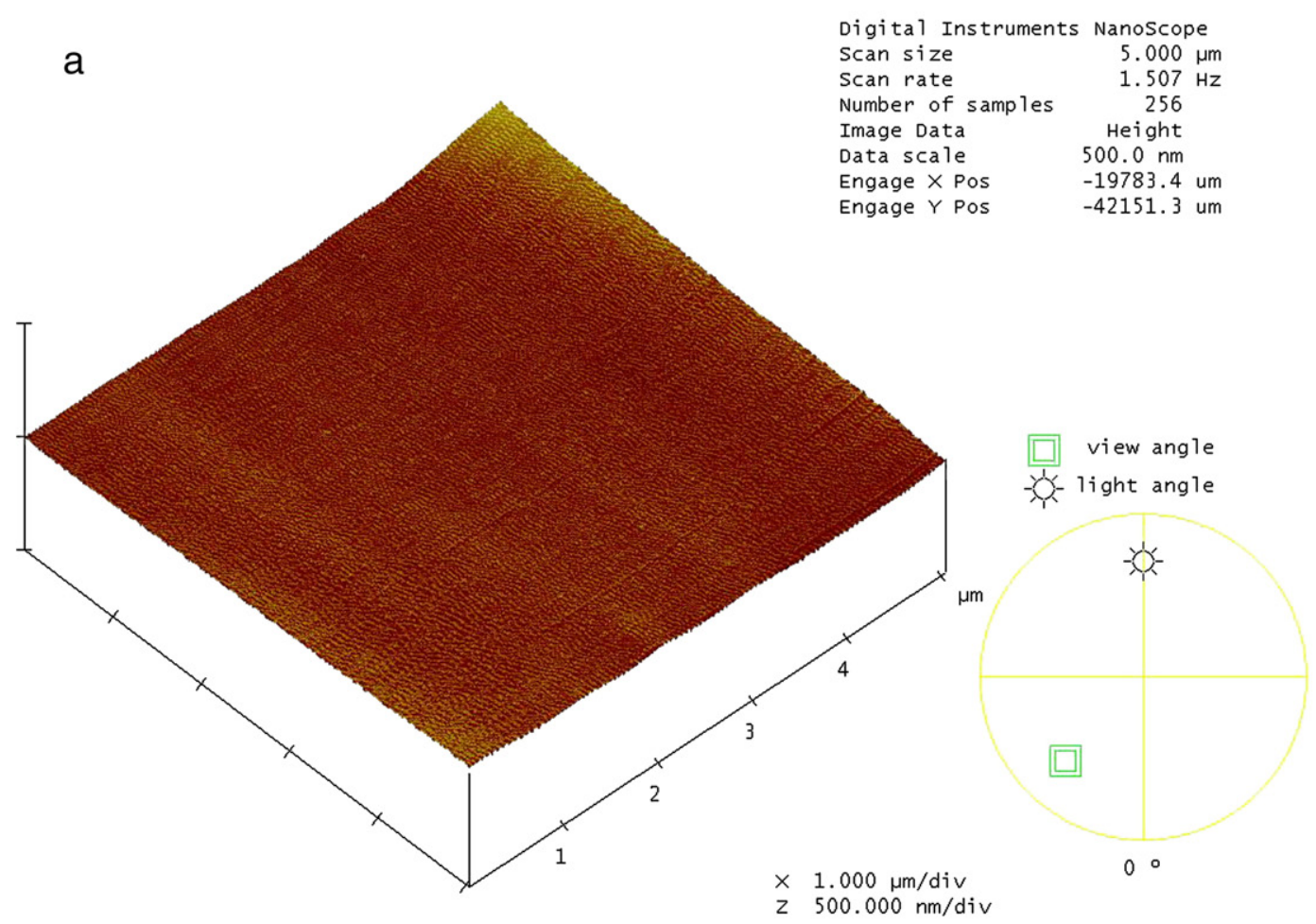

$3 \mathrm{dcbr} 1$

b
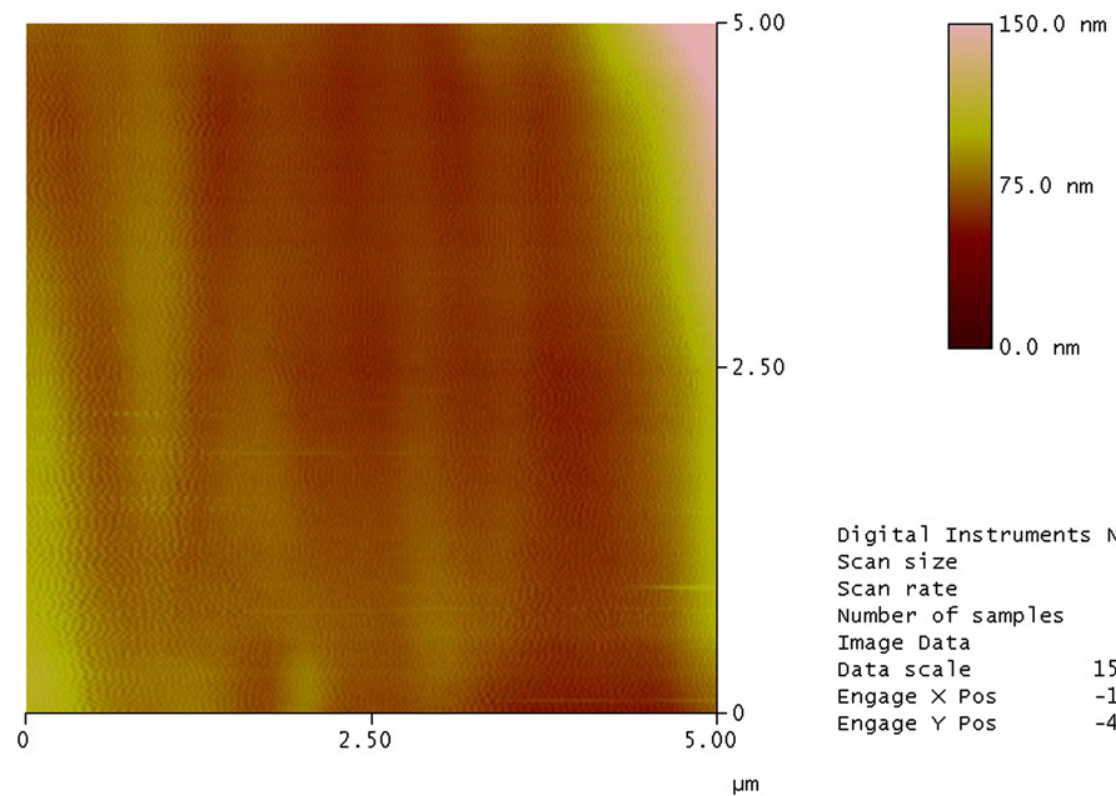

Digital Instruments NanoScope Scan size $\quad 5.000 \mu \mathrm{m}$ Scan rate $\quad 1.507 \mathrm{~Hz}$ Number of samples 256 Image Data Height Data scale $\quad 150.0 \mathrm{~nm}$ Engage $X$ Pos $\quad-19783.4$ um $\begin{array}{ll}\text { Engage } X \text { Pos } & -19783.4 \text { um } \\ \text { Engage } Y \text { Pos } & -42151.3 \text { um }\end{array}$ 
This outward-directed force is caused by the dispersive force due to the capillary action of the pinned bR droplet that exists towards the edge of the bR droplet when allowed to dry normally. As more and more material was dispersed to the surrounding sides, the centre becomes visually void when completely dried. As such, the overcompressed bR layer at the edge of the void centre as shown in the profile in Photomicrograph 1 clearly demonstrates the effect of the dispersion force resulting in the "coffee-ring" effect.

The electric field assisted sample meanwhile had a visibly compressed centre as shown in Photomicrograph 2. The bulk of material in this case seems to converge to the centre as compared to the non-electric field assisted bR film. Uniform electric field distribution allows uniform distribution of the bR molecules and as a result no void centre was seen. Visually smoother profiles were observed in the AFM pictures shown in Picture 2, justifying the "central" smoothness obtained under the electric field induced bR film formation (Figs. 3 and 4).

The existence of the centralized and compressed region may be due to slower overall dispersion (under the strong electric field) as compared to a higher drying rate. It is also known that the negatively charged bR molecules may stay immobile as a result of being attracted to the positively biased ITO slide. Such effect also accounts for the over compressed central region when dried under the strong electric field. Therefore, the bR droplet dries-up at a faster rate leaving the bulk of the material still in the centre as observed in Photomicrograph 2.

\subsection{Fractal morphological analysis of $b R$ distribution}

Fractal methods have become standard tools to analyze and characterize surface morphologies in material science [24] and geophysics Turcotte [25]. One of the most commonly determined morphological parameters from fractal analysis is the fractal dimension, $D$. Another closely related parameter is the surface roughness index, also known as the local self-similar or Hurst exponent $H$ that can be measured from the scaling behavior of the surface height. These two parameters are related by $D=2-H$ for intersect profiles and $D=3-H$ for surface realizations. Power-law scaling of the covariance function in the form of $C(\tau) \sim|\tau|^{-\beta}$, for large (time or spatial) lag $\tau$ with correlation exponent $\beta$, is another feature of fractal processes that are profound in many diverse phenomena. A process is said to exhibit long-range dependence (LRD) if $0<\beta<1$. If one chooses to model LRD process using the increments of the FBM or the fractional Gaussian noise, then correlation exponent is related to the Hurst exponent through $\beta=2-2 \mathrm{H}$ $[26,27]$. As mentioned earlier, FBM model ties down the relationship between fractal dimension and the Hurst exponent thus cannot be used to characterize local regularity and LRD properties, independently. An alternative model that allows independent characterization of these two features is the class of generalized Cauchy processes suggested in $[17,28]$. For the modeling of two-dimensional data or images, consider GCP as a stationary isotropic Gaussian field with constant mean (may be assumed to be zero) and covariance function given by

$C(h)=\frac{\sigma^{2}}{\left(1+|| h \|^{\alpha}\right)^{\beta / \alpha}}, \quad h \in R^{2}$,

where $0<\alpha \leq 2, \beta>0$ and $\sigma^{2}$ is the variance of the process. The semivariogram is then defined as

$$
\begin{aligned}
\gamma(h) & =[C(0)-C(h)] \\
& =\sigma^{2}\left(1-\frac{1}{\left(1+|| h||^{\alpha}\right)^{\beta / \alpha}}\right) .
\end{aligned}
$$

Asymptotic behaviors of Eq. (1) is given by

$C(h)= \begin{cases}\left.|| h\right|^{\alpha}, & || h|| \rightarrow 0 \\ || h||^{-\beta}, & || h|| \rightarrow \infty\end{cases}$

Exponents $\alpha$ and $\beta$ control the fractal scaling and LRD properties, respectively. Two-dimensional surface realization of GCP has fractal dimension of $D=3-\alpha / 2$, with $0<\alpha \leq 2$ and it is said to exhibits longrange dependence (or strong spatial correlation) if $0<\beta<1$. Correlation exponent $\beta$ is also related the Hurst exponent via $H=1-\beta / 2$. Two-dimensional GCP can be simulated using Fourier spectral method with the knowledge of the covariance. Surface realization of the process for two different sets of model parameters are shown in Fig. 1. LRD surfaces $(0<\beta<1)$ shows large scale aggregates and larger values of $\alpha$ produce smoother textures. With GCP, these two parameters can be varied independently.

In this study, we considered gray-level images with intensities $Z$ ( $x$, $y$ ) at grid points $(j, k)=\left(x_{j}, y_{k}\right), j, k=1, \ldots, M$; with $M^{2}$ is the total number of the points on the surface. Five samples of square images of size $(128 \times 128)$-pixels are clipped from the original images of bR with and without electric field. Estimator for the two-dimensional empirical semi-variogram is then defined as [29] (Fig. 5),

$\hat{\gamma}\left(h_{x}, h_{y}\right)=\frac{1}{\left[\left(M-h_{x}\right)\left(M-h_{y}\right)\right]} \sum_{j=1}^{M-h_{x}} \sum_{k=1}^{M-h_{y}}\left(Z\left(j+h_{x}, k+h_{y}\right)-Z(j, k)\right)^{2}$

where $M-h_{x}, M-h_{y}$ are the number of pairs of points, respectively at a lag distance $h_{x}, h_{y} \in[-M / 2, M / 2]$. If the surface is isotropic then the

\section{a}

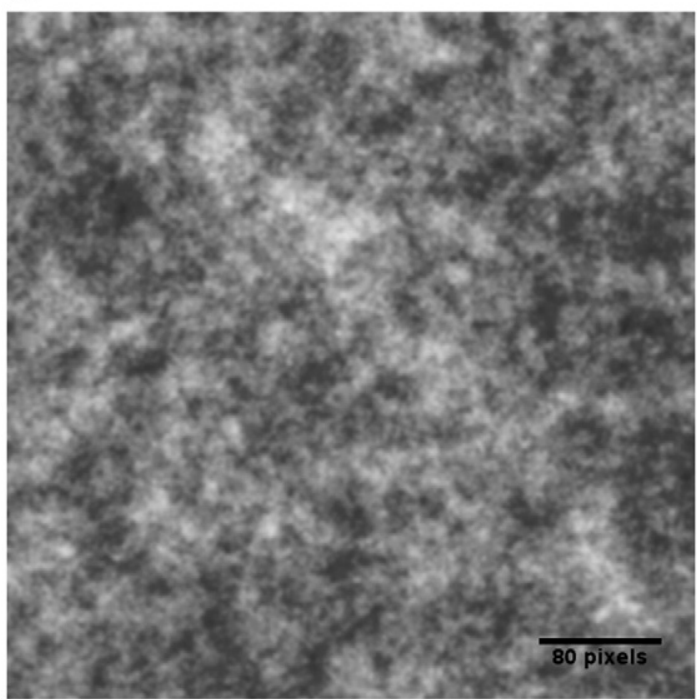

b

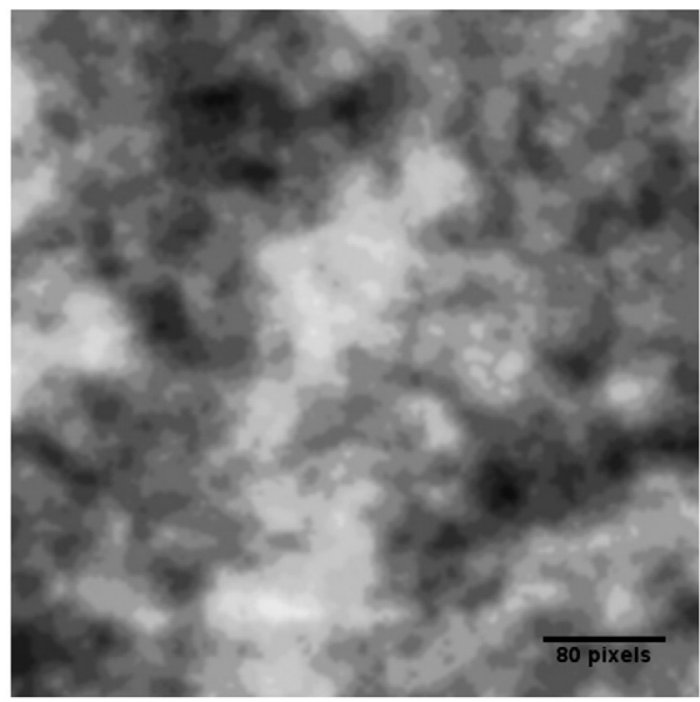

Fig. 5. Surface realizations of generalized Cauchy processes for model parameters (a) $\alpha=0.85, \beta=1.75$ and (b) $\alpha=1.75, \beta=0.85$. 


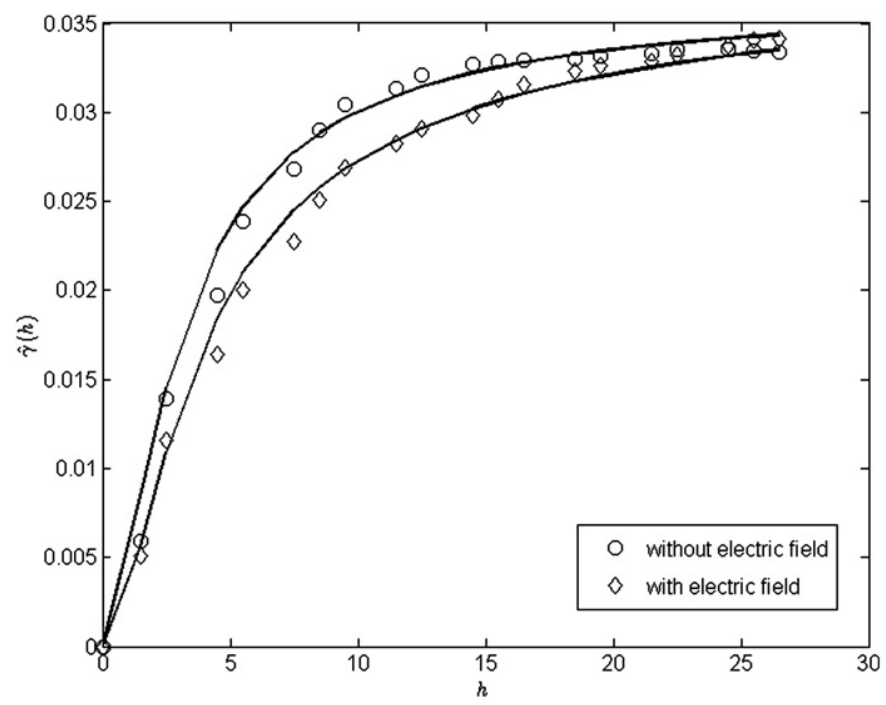

Fig. 6. Empirical semi-variograms and the least square fitted model covariances based on generalized Cauchy processes.

two-dimensional semi-variogram can be reduced to one-dimensional case $\hat{\gamma}(h)$, where $h=\sqrt{h_{x}^{2}+h_{y}^{2}}$. The empirical semi-variograms are fitted using ordinary least square technique with semi-variogram of the GCP given in Eq. (2) with model parameters $\left\{\alpha, \beta, \sigma^{2}\right\}$. We obtained $\left\{\alpha=1.84 \pm 0.03, \beta=0.867 \pm 0.025, \sigma^{2}=0.040 \pm 0.001\right\}_{\mathrm{E}}$ for the morphologies of bR with electric field and $\{\alpha=1.55 \pm 0.02$, $\left.\beta=1.23 \pm 0.010, \sigma^{2}=0.036 \pm 0.001\right\}$ for the condition where the electric field is absent. Fractal dimension and the Hurst exponent are then estimated to be $\{D, H\}_{\mathrm{E}}=\{2.08 \pm 0.03,0.567 \pm 0.016\}$ and $\{D$, $H\}_{\mathrm{NE}}=\{2.23 \pm 0.01,0.386 \pm 0.002\}$ for $\mathrm{bR}$ with and without external electric field, respectively (Fig. 6).

The empirical semi-variograms are shown in Fig. 2 with their respective least square fitting of the generalized Cauchy process semivariograms. The analysis also supported visual inspection that the distribution of the bR protein is smoother and exhibits strong spatial correlation in the presence of electric field.

\section{Conclusions}

In our present work, there was a need to control the "coffee-ring" effect so as to prepare naturally air-dried uniform bR layers. The applied directional electric field were also meant as an external tool to orientate the dipole moment of the uniformly spread bR molecules [23]. Such orientation enables efficient photoresponse when incorporated into the fabrication of bR-based sensors.

Placing the bR loaded ITO slide electrically connected to the positive aluminium electrode practically inhibited the outward dispersion of the negatively charged bR molecules. Therefore, by simply applying electric field during film formation, a good amount of control was successfully demonstrated in eliminating the formation of the "coffee-ring" effect in this work.

We also performed fractal analysis of the surface morphologies of the bR layers to investigate local distribution of bR protein and their spatial correlation. In the presence of external electric field, surface morphologies are smoother with long-range correlation, which justifies the uniform aggregation towards closing up the void. Further works into the possibility of precisely controlling the inhibition rate of the ring formation by characterizing the electric field factor may openup many practical technological possibilities in printing and coating processes.

\section{Acknowledgments}

This work was financially supported by the VOTE-F grant IPP/ UPDiT/Geran (PJP)/F0161/2003D, University of Malaya and the SAGA Grant 66-02-03-0083, Academy of Sciences Malaysia.

\section{References}

[1] D. Oesterhelt, Angew. Chem. Int. Ed. Engl 15 (1976) 316.

[2] D. Oesterhelt, W. Stoeckenius, Nat. New Bio. 233 (1971) 149.

[3] R.H. Lozier, R.A. Bogomolni, W. Stoeckenius, Biophys. J. 15 (1975) 955.

[4] T. Miyasaka, K. Koyama, Appl. Opt. 32 (1993) 6371.

[5] Q. Ren, Y.P. Zhao, L. Han, H.B. Zhao, Nanotech. 17 (2006) 1778.

[6] J.P. Sharkany, S.O. Korposh, Z.I. Batori-Tarci, I.I. Trikur, J.J. Ramsden, Sens. Actuators B 107 (2005) 77.

[7] T. Renner, N. Hampp, Opt. Comm. 96 (1993) 142.

[8] P. Vengadesh, A.B. Wan Haliza, S. Anandan, K.S. Low, Org. Elect. 17 (2006) 300.

[9] H.G. Choi, W.C. Jung, J. Min, W.H. Lee, J.W. Choi, Biosens. Bioelect. 16 (2001) 925

[10] J. Min, H.G. Choi, J.W. Choi, W.H. Lee, U.R. Kim, Thin Solid Films 327-329 (1998) 698.

[11] H. Hu, R.G. Larson, J. Phys. Chem. B Lett. 110 (2006) 7090.

[12] H. Kaya, B. Jerome, P. Colinet, Europhys. Lett. 74 (5) (2006) 861.

[13] R.D. Deegan, O. Bakajin, T.F. Dupont, G. Huber, S.R. Nagel, F.A. Witten, Nature 389 (1990) 827.

[14] R.D. Deegan, O. Bakajin, T.F. Dupont, G. Huber, S.R. Nagel, F.A. Witten, Phys. Rev. E62 (2000) 756.

[15] F. Kienberger, C. Stroh, G. Kada, R. Moser, W. Baumgartner, V. Pastushenko, C. Rankl, U. Schmidt, H. Muller, E. Orlova, C. LeGrimellec, D. Drenckhahn, D. Blaas, P. Hinterdorfer, Ultramicroscopy 97 (2003) 229.

[16] D.T. Kim, H.W. Blanch, C.J. Radke, J. Colloids Surf. B, Biointerfaces 41 (2005) 263.

[17] T. Gneiting, J. Appl. Probab. 37 (4) (2000) 1104.

[18] B.B. Mandelbrot, The Fractal Geometry of Nature, Freeman, San Francisco, 1982

[19] S.C. Lim, M. Li, J. Phys. Maths. A: Math. Gen. 39 (2006) 2935.

[20] A. Tscheschel, D. Stoyan, J. Lacayo, J. Microscopy 217 (2005) 75.

[21] H.A. Makse, S. Havlin, M. Schwartz, H.E. Stanley, Phys. Rev. E53 (5) (1996) 5445.

[22] A.A. Kononenko, E.P. Lukashev, S.K. Chamorovsky, et al., Biochim. Biophys. Acta 862 (1987) 56.

[23] C. Nicolini, V. Erokhin, S. Paddeu, C. Paternolli, M.K. Ram, Biosens. Bioelect. 4 (1999) 427.

[24] F. Family, T. Viscek, Dynamics of Fractal Surfaces, World Scientific, Singapore, 1991

[25] D. Turcotte, Fractals and Chaos in Geology and Geophysics, Cambridge University Press, Cambridge, 1997.

[26] B.B. Mandelbrot, J. Van Ness, J. SIAM Rev. 10 (4) (1968) 422

[27] J. Beran, Statistics for Long Memory Processes, Chapman \& Hall, New York, 1994.

[28] T. Gneiting, M. Schlather, SIAM Rev. 46 (2) (2004) 269.

[29] N.A.C. Cressie, Statistical for Spatial Data, John Wiley \& Sons, New York, 1993. 